\title{
Clinical validation of the next-generation sequencing-based Extended RAS Panel assay using metastatic colorectal cancer patient samples from the phase 3 PRIME study
}

\author{
Nitin Udar ${ }^{1}$ (1) Catherine Lofton-Day ${ }^{2} \cdot$ Jun Dong ${ }^{3} \cdot$ Darcy Vavrek $^{4} \cdot$ A. Scott Jung ${ }^{5} \cdot$ Kristen Meier $^{4} \cdot$ Anita lyer $^{1}$. \\ Ryan Slaughter ${ }^{6} \cdot$ Karen Gutekunst $^{1} \cdot$ Bruce A. Bach $^{7} \cdot$ Marc Peeters $^{8} \cdot$ Jean-Yves Douillard ${ }^{9}$
}

Received: 9 March 2018 / Accepted: 13 June 2018 / Published online: 17 July 2018

(c) The Author(s) 2018

\begin{abstract}
Purpose To validate a next-generation sequencing (NGS)-based companion diagnostic using the MiSeqDx ${ }^{\circledR}$ sequencing instrument to simultaneously detect 56 RAS mutations in DNA extracted from formalin-fixed paraffin-embedded metastatic colorectal cancer (mCRC) tumor samples from the PRIME study. The test's ability to identify patients with mCRC likely to benefit from panitumumab treatment was assessed.

Methods Samples from PRIME, which compared first-line panitumumab + FOLFOX4 with FOLFOX4, were processed according to predefined criteria using a multiplex assay that included input DNA qualification, library preparation, sequencing, and the bioinformatics reporting pipeline. NGS mutational analysis of $K R A S$ and $N R A S$ exons 2,3, and 4 was performed and compared with Sanger sequencing.

Results In 441 samples, positive percent agreement of the Extended RAS Panel with Sanger sequencing was $98.7 \%$ and negative percent agreement was 97.6\%. For clinical validation $(n=528)$, progression-free survival (PFS) and overall survival (OS) were compared between patients with $R A S$ mutations ( $R A S$ Positive) and those without (RAS Negative). Panitumumab + FOLFOX4 improved PFS in $R A S$ Negative patients $(P=0.02)$. Quantitative interaction testing indicated the treatment effect (measured by the hazard ratio of panitumumab + FOLFOX4 versus FOLFOX4) differed for RAS Negative versus RAS Positive for PFS $(P=0.0038)$ and OS $(P=0.0323)$.

Conclusions NGS allows for broad, rapid, highly specific analyses of genomic regions. These results support use of the Extended RAS Panel as a companion diagnostic for selecting patients for panitumumab, and utilization is consistent with recent clinical guidelines regarding mCRC RAS testing. Overall, approximately $13 \%$ more patients were detected with the Extended RAS Panel versus KRAS exon 2 alone.

Clinical trial registry identifier NCT00364013 (ClinicalTrials.gov).
\end{abstract}

Keywords Gastrointestinal cancers $\cdot$ Colorectal $\cdot$ New software for data analysis $\cdot$ Mutation detection methods $\cdot$ Molecular diagnosis and prognosis

\section{Introduction}

Electronic supplementary material The online version of this article (https://doi.org/10.1007/s00432-018-2688-3) contains supplementary material, which is available to authorized users.

Nitin Udar

greatbioinformatics@yahoo.com

Extended author information available on the last page of the article
Molecular genetic studies targeting metastatic colorectal cancer (mCRC) tumors have identified mutations in KRAS and NRAS that predict a lack of therapeutic response to epidermal growth factor receptor (EGFR) inhibitors (Bokemeyer et al. 2015; Douillard et al. 2013; Peeters et al. 2013; Van Cutsem et al. 2015). As a result, the European Society for Medical Oncology (ESMO) (Van Cutsem et al. 2016), the National Comprehensive Cancer Network (NCCN) (National Comprehensive Cancer Network 2017), and the American Society for Clinical Pathology, the College of American 
Pathologists, the Association for Molecular Pathology, and the American Society of Clinical Oncology recommended extended RAS mutation testing of KRAS and NRAS exon 2 (codons 12 and 13), exon 3 (codons 59 and 61), and exon 4 (codons 117 and 146) to improve patient selection for anti-EGFR treatment, thereby improving patient outcomes (Sepulveda et al. 2017).

PRIME (20050203) was a phase 3, multicenter, openlabel, randomized trial that evaluated the treatment effect of panitumumab plus FOLFOX4 compared with FOLFOX4 alone as first-line therapy in 1183 patients with wild-type KRAS exon 2 mCRC (Douillard et al. 2010; Peeters et al. 2012). PRIME has been analyzed sequentially as additional information about activating $R A S$ mutations has become available. Archived CRC tissue samples were most commonly isolated from the primary lesions of patients with mCRC. These samples were tested in a prospective-retrospective analysis for additional activating $R A S$ mutations beyond $K R A S$ exon 2 using Sanger sequencing. The ascertainment rate of tumor $R A S$ status was $90 \%$ ( $n=1060 / 1183$ ). In-depth sequencing identified an additional $17 \%$ of samples that carried $R A S$ mutations in addition to those originally identified in KRAS exon 2. These mutations predicted a lack of response to EGFR inhibition in patients who received panitumumab plus FOLFOX4. Moreover, patients treated with panitumumab plus FOLFOX4 with $R A S$ mutations had worse progression-free survival (PFS; 7.3 months) and overall survival (OS; 15.5 months) compared with those who had no RAS mutations (PFS, 10.1 months; OS, 25.8 months) (Douillard et al. 2013).

Next-generation sequencing (NGS) has emerged as a powerful tool that provides broad, rapid, highly specific analyses of genomic regions of interest in a single assay (Shendure and Ji 2008). Because NGS has the capability to interrogate millions of DNA fragments in parallel, this technology decreases sequencing time, labor, and reagents, which significantly reduces cost and time to results compared with iterative single locus testing. The Extended RAS Panel is a United States Food and Drug Administrationapproved, qualitative in vitro diagnostic NGS test developed for the simultaneous detection of 56 known activating mutations within exons 2, 3, and 4 of the KRAS and NRAS genes. The assay system includes sequence-specific reagents and consumables for DNA qualification, library preparation, and sequencing, as well as integrated data analysis and reporting software. The assay is intended for use with the MiSeqDx ${ }^{\circledR}$ (Illumina, Inc., San Diego, CA, USA) sequencing instrument. The goal of this analysis was to clinically validate a companion diagnostic (CDx) test to aid in the identification of patients with mCRC eligible for treatment with panitumumab (Vectibix ${ }^{\circledR}$, Amgen Inc., Thousand Oaks, CA, USA).

The clinical accuracy of the Extended RAS Panel was evaluated by comparing the results provided by the NGS assay to those of the reference method, Sanger bidirectional sequencing. For the clinical validation study, clinical outcomes were examined to determine if there was an improvement in PFS and OS in wild-type RAS patients treated with panitumumab plus FOLFOX4 versus FOLFOX4 alone when $R A S$ status was determined by the Extended RAS Panel.

\section{Materials and methods}

\section{Patients}

Patient eligibility criteria for the randomized controlled PRIME study have been previously described (Douillard et al. 2010).

\section{Pathology assessment}

All randomized patients with available and eligible formalin-fixed paraffin-embedded (FFPE) samples were evaluated by a board-certified pathologist at a Clinical Laboratory Improvement Amendments (CLIA)-certified laboratory. Microscopic inspection of hematoxylin and eosin stained slides was performed to ascertain the area of the tissue and the tumor. Only samples meeting a $\geq 50 \%$ tumor content were selected for DNA extraction. Samples containing $<50 \%$ tumor tissue were enriched using macrodissection to remove normal tissue content. For optimal yield of amplifiable DNA, the recommended cumulative tissue area was $\geq 240 \mathrm{~mm}^{2}$ (at least $8 \times 5 \mu \mathrm{M}$ serial sections). The results of the pathology review were recorded, and qualified samples were processed for DNA extraction by an external, independent CLIA-certified laboratory for RAS CDx testing and a second CLIA-certified laboratory for Sanger sequencing. Individuals involved in the $R A S$ sample laboratory testing did not have access to treatment allocation or study clinical outcomes.

\section{Sample qualification, library preparation, and sequencing}

The assay uses a dual strand approach using TruSeq Custom Amplicon (TSCA) technology (Illumina, Inc., San Diego, CA, USA) to distinguish true mutations from artifacts commonly found in DNA from FFPE tissue. Following DNA extraction using the QIAGEN FFPE extraction kit (QIAGEN, Germantown, MD, USA), the samples were prepared for NGS. The Extended RAS Panel assay involves three main steps (Fig. 1). The first step is to qualify the DNA sample to be used for the assay. Extracted DNA samples are subjected to quantitative polymerase chain reaction (qPCR), which assesses the sample's quality and quantity, or amplifiability. The samples are qualified by measuring amplifiability 


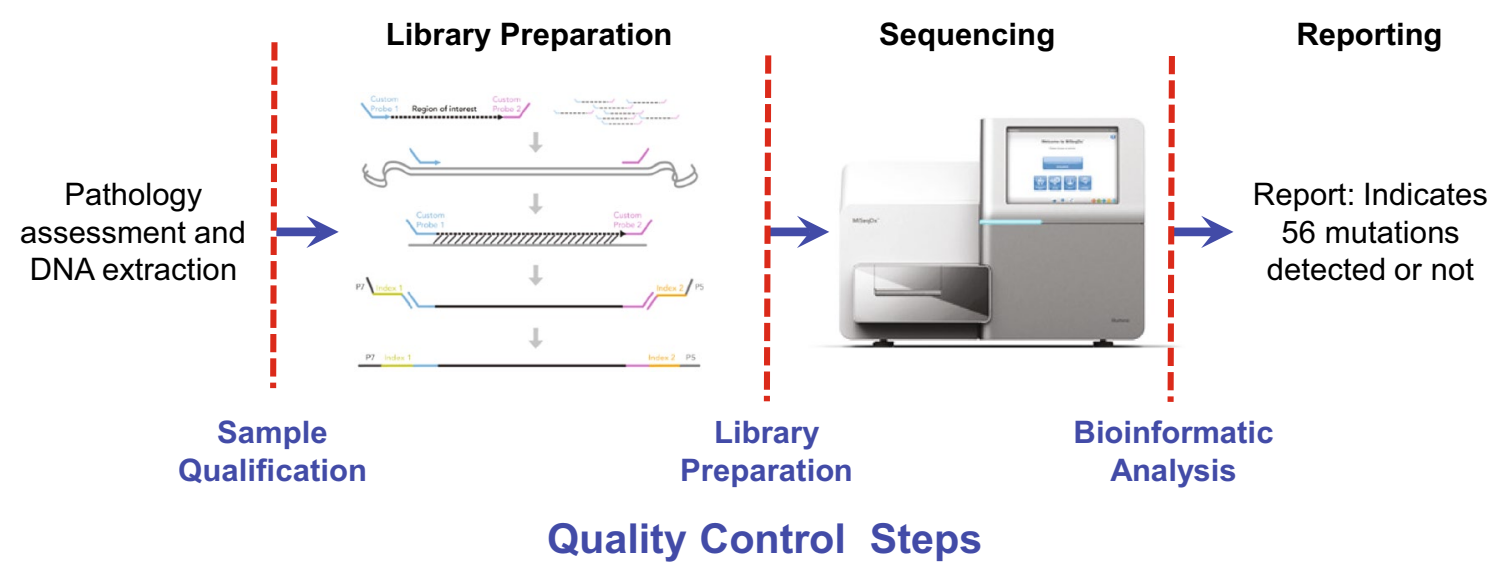

Fig. 1 Extended RAS Panel assay workflow

relative to a control DNA template. The metric to assess the quality of FFPE DNA is the change in quantification threshold cycle $(\mathrm{dCq})$ between sample and control DNA. If this $\mathrm{dCq}$ value is $\leq 5.0$, the samples are eligible to advance to step two, library preparation. A dCq level of -0.5 to 5.0 corresponds to approximately $1200 \mathrm{ng}$ to $25 \mathrm{ng}$, respectively, of intact DNA used in the assay.

The second step is to prepare the samples for sequencing. Library preparation using TSCA consists of four key steps: hybridization, extension-ligation, PCR amplification, and library normalization. In hybridization, oligonucleotide probes targeting specific $R A S$ mutations are hybridized to the sample genomic DNA (each DNA strand is independently targeted by two oligonucleotide probe pools). Extension-ligation connects the hybridized upstream and downstream oligonucleotides to form products that contain the $R A S$-specific oligonucleotides flanked by sequences required for amplification. PCR amplification amplifies the extension-ligation products using primers that add index sequences for multiplexing of numerous patient samples in a single assay, as well as common adapters required for cluster generation on the MiSeqDx sequencing instrument. Following library preparation, as a quality control (QC) measure, the samples are electrophoresed on an agarose gel to visualize the amplified product (ie, the library). At the end of this process, a PCR clean-up procedure purifies the PCR products (referred to as a library). Library normalization balances the quantity of each library to ensure equal library representation in the final pooled library. Following this step, the pooled library is loaded onto the MiSeqDx sequencing instrument for sequencing using sequencing by synthesis (SBS) chemistry. Up to 10 samples and two controls (positive and negative) can be sequenced together.

After sequencing is completed, the instrument software analyzes the data. During this analysis, sequencing reads can be traced back to their unique originating source sample based on their index sequence (ie, demultiplexing). Several quality parameters are assessed and a final report detailing whether any mutations are identified is generated.

The positive and negative controls are prepared in parallel with the samples and are included in every sequencing run. The positive control consists of two RAS panel mutations that have low allele frequency and is used to evaluate the performance of the library preparation and sequencing. The panel control must generate the expected genotype to be valid. If the control is invalid, processing errors may have occurred. The software will fail the entire sequencing run and all samples will appear as invalid. The negative control serves to detect issues like cross-contamination. If an unexpected outcome occurs in the negative control, the software will fail the entire sequencing run and all samples will appear as invalid.

Only results for the 56 mutations listed in Table S1 are reported. The panel mutations were selected based on four panitumumab clinical sequencing studies (Douillard et al. 2010; Patterson et al. 2013; Peeters et al. 2015; Schwartzberg et al. 2014), the Catalogue Of Somatic Mutations In Cancer (COSMIC) database, the NCCN guidelines (National Comprehensive Cancer Network 2017), and the Vaughn et al. publication (2011). For calculation of clinical accuracy, results from the Extended RAS Panel testing were compared with the output from the antecedent Sanger sequencing.

In the primary analysis of the PRIME study as reported by Douillard et al. (2010), the therascreen investigational use only PCR kit (QIAGEN) was used to identify KRAS exon 2 mutations by analyzing seven somatic mutations in $K R A S$ codons 12 and 13 (ie, alleles G12A, G12D, G12R, G12C, G12S, G12V, or G13D). Tumor samples from patients identified as having one or more of the seven KRAS exon 2 mutations and with sufficient additional tissue sections that met previously described quality criteria underwent additional testing for mutations in exons 2, 3, and 4 of KRAS and NRAS 
using Sanger bidirectional sequencing. Samples identified to have no mutations in KRAS exon 2 as determined by the therascreen assay were also tested for other mutations in exon 2 of KRAS. These extended Sanger sequencing data, which included KRAS exons 3 and 4 and NRAS exons 2, 3, and 4 status, were compiled with Sanger data from Douillard et al. (2013) to provide complete exon coverage for the qualified PRIME samples (Douillard et al. 2013).

Both the Extended RAS Panel and Sanger sequencing provided qualitative assessments of mutation status for both $K R A S$ and NRAS. After passing predefined quality parameters to meet sequencing validity requirements, the final result of each method was a binary qualitative assignment into either $R A S$ Mutation Detected (RAS Positive) or RAS Mutation Not Detected (RAS Negative) based on the identification of any of the 56 panel mutations. For NGS, a RAS Positive result was assigned if at least one $R A S$ panel mutation was detected, a $R A S$ Negative result was assigned if no $R A S$ panel mutations were detected, and an invalid result was assigned if the data from a sample or a sequencing run were of insufficient quality. For Sanger sequencing, a $R A S$ Positive result was assigned if a $R A S$ mutation was detected in at least one exon, a RAS Negative result was assigned if no RAS mutations were detected in any exons, and an invalid result was assigned if at least one exon had an invalid result and others were either wild-type or invalid.

After Extended RAS Panel testing was completed, the results (mutation detected or mutation not detected) were linked to the most recent clinical endpoints for PFS and OS (data cutoffs for PFS and OS were 2010 and 2013, respectively) from the PRIME study data.

\section{Statistical analysis}

Accuracy was assessed by calculating the positive percent agreement (PPA) and negative percent agreement (NPA) between the Extended RAS Panel and Sanger sequencing utilizing the patient-level overall $R A S$ results.

The goal of the clinical validation statistical analysis reported here was to demonstrate whether there was an improvement in PFS and OS in patients treated with panitumumab plus FOLFOX4 versus FOLFOX4 alone whose tumors were found to have none of the 56 RAS mutations as determined by the Extended RAS Panel (RAS Negative Analysis Set). All hypotheses tested and confidence intervals (CIs) described were two-sided unless otherwise stated. A $5 \%$ significance level was used to compare the treatment effect on PFS and OS in the RAS Negative Analysis Set in a sequential manner. Tests of PFS and OS on all efficacy analysis sets were considered descriptive. All $P$ values were not corrected for multiplicity and were considered descriptive.

The efficacy analyses of PFS and OS focused on the most recent clinical endpoints, and disease assessment was based on blinded central review of imaging studies using modified Response Evaluation Criteria in Solid Tumors criteria. Time-to-event variables were summarized using hazard ratios (HRs), Kaplan-Meier (KM) curves, KM estimates for quartiles, and log-rank test $P$ values. Point estimates with 95\% CIs were calculated.

Quantitative interaction tests were performed to evaluate whether the treatment effect as measured by the HR (panitumumab plus FOLFOX4:FOLFOX4 alone) was the same in $R A S$ Negative patients versus $R A S$ Positive patients. Interaction was estimated as the ratio of the HR for the $R A S$ Negative Analysis Set over the HR for the mutant RAS Positive Analysis Set using methods from Gail and Simon to determine the magnitude and direction of the effect size in the biomarker-identified populations (Gail and Simon 1985).

\section{Results}

\section{Patients}

Of 1183 randomized patients, 891 had valid Sanger sequencing results, 528 had valid NGS results with a $\mathrm{dCq} \leq 5.0$, and 441 had both Extended RAS Panel and Sanger results and were used for the accuracy analysis. Patients were not included for the following reasons: 358 (30.3\%) had no available remaining tissue for $R A S$ testing, $127(10.7 \%)$ had insufficient tissue area, 13 (1.1\%) had insufficient neoplastic area, 215 (18.2\%) had DNA of insufficient quality (214 had a $\mathrm{dCq}>5.0$ and one had a nonestimable $\mathrm{dCq}$ value), 28 (2.4\%) were QC failures (including gel check failures and sequencing QC invalids; samples with invalid gel results were repeated using a second library preparation), and one $(0.08 \%)$ had sample processing errors. To determine whether there was bias introduced by the truncation in the number of samples available using both methods, baseline disease characteristics for the 441 patients were compared with those of the RAS Unevaluable Set (Table S2). There were no apparent differences between cases with available tissue and those without available tissue.

\section{Accuracy}

Of the 441 patients with valid Extended RAS Panel and Sanger results, 211 (48\%) were RAS Negative and 230 (52\%) were RAS Positive by Sanger sequencing. Of the $230 R A S$ Positive patients, 227 were $R A S$ Positive by the Extended RAS Panel [PPA $=98.7 \%(n=227 / 230) ; 95 \%$ CI 96.2-99.7\%]. Of the $211 R A S$ Negative patients by Sanger, 206 were $R A S$ Negative by the Extended RAS Panel [NPA $=97.6 \%(n=206 / 211) ; 95 \%$ CI 94.6-99.2\%; Table 1]. The lower limit of the 2-sided 95\% CI (exact Clopper-Pearson) for both PPA and NPA exceeded $90 \%$, and therefore the 
Table 1 Positive and negative percent agreement of patient-level $R A S$ results $(\mathrm{dCq} \leq 5.0)$

\begin{tabular}{cccc}
\hline \multirow{2}{*}{$\begin{array}{c}\text { MiSeqDx Extended RAS } \\
\text { Panel }\end{array}$} & Positive & Sanger & Total \\
\cline { 2 - 4 } Positive & $227^{\mathrm{a}}$ & 5 & 232 \\
Negative & $3^{\mathrm{b}}$ & 206 & 209 \\
Total & 230 & 211 & 441 \\
\hline & Performance Summary & $\mathbf{9 5 \%}$ CI \\
\hline Agreement Concordance & Point Estimate & $96.2 \%-99.7 \%$ \\
Positive percent agreement & $227 / 230=98.7 \%$ & $94.6 \%-99.2 \%$ \\
\hline Negative percent agreement & $206 / 211=97.6 \%$ & \\
\hline
\end{tabular}

$\mathrm{CI}=$ confidence interval.

${ }^{\text {a }}$ There were 224 exact matches for within-patient all mutation level results; for two patients, the Extended RAS Panel detected two mutations, whereas Sanger detected one mutation; one patient had different mutations detected by MiSeqDx and Sanger.

${ }^{\mathrm{b}}$ One patient had two mutations detected by Sanger; two patients had one mutation detected by Sanger.

acceptance criteria were met. Eleven patients had conflicting results between Sanger sequencing and the Extended RAS Panel analysis; however, only eight patients had discrepant patient-level results (Table S3).

\section{Clinical validation}

For patients whose tumors were RAS Negative, the median PFS time was 10.0 months for the panitumumab plus FOLFOX4 group and 9.2 months for the FOLFOX4 alone group. The HR (panitumumab plus FOLFOX4:FOLFOX4 alone) for PFS from a stratified Cox proportional hazards model was 0.700 (95\% CI 0.516-0.948), indicating longer PFS for the panitumumab plus FOLFOX4 group than for the FOLFOX4 alone group. The stratified log-rank test revealed a statistically significant difference in PFS between the groups $(P=0.0206)$. For patients whose tumors were RAS Positive, the HR for PFS was 1.242 (95\% CI 0.976-1.582), indicating longer PFS for the FOLFOX4 alone group than for the panitumumab plus FOLFOX4 group. Quantitative interaction testing indicated the treatment effect differed between patient groups, as measured by panitumumab plus FOLFOX4 versus FOLFOX4 alone, when comparing RAS Negative patients with RAS Positive patients $(P=0.0038$; Table 2$)$.

For $R A S$ Negative patients, the median OS time was 26.0 months for the panitumumab plus FOLFOX4 group and 23.1 months for the FOLFOX4 alone group. The HR for OS from a stratified Cox proportional hazards model was 0.754 (95\% CI $0.555-1.024 ; P=0.0694)$, indicating longer survival for the panitumumab plus FOLFOX4 group than for the FOLFOX4 alone group, but it was not statistically significant. For RAS Positive patients, the HR was 1.156 (95\% CI 0.906-1.476), indicating longer OS for the FOLFOX4 alone group than for the panitumumab plus FOLFOX4 group. Quantitative interaction testing revealed a statistically significant difference in OS $(P=0.0323$; Table 2). Kaplan-Meier plots for PFS and OS in RAS Negative patients and $R A S$ Positive patients are shown in Fig. 2a, b.

For the KRAS exon 2 Negative/RAS Positive subset (patients who did not have a mutation in $K R A S$ exon 2, but did have a mutation in KRAS exons 3 or 4 or NRAS exons 2, 3 , or 4), the trends were in the unfavorable direction: PFS and OS were longer in the FOLFOX4 alone group compared with the panitumumab plus FOLFOX4 group (Table 3).

The safety profile for panitumumab in patients with $R A S$ Negative mCRC was similar to that in patients with KRAS exon 2 Negative mCRC.

\section{Mutation distributions}

The overall prevalence of $R A S$ mutations is shown in Table S4. Of all the mutations detected by the Extended RAS Panel $(n=311)$, mutations in KRAS exons 2,3 , and 4 occurred in $81.7,4.2$, and $6.1 \%$ of samples, respectively, and mutations in NRAS exons 2, 3, and 4 occurred in 2.6, 5.5 , and $0 \%$ of detected mutations, respectively (Fig. S1, supplement).

\section{Mutation frequencies}

Using $\geq 50 \%$ tumor content in the FFPE specimens, the distribution of mutation frequencies observed in the PRIME study for samples that tested positive is shown in Fig. 3. The majority of specimens showed mutation frequencies in the $0.15-0.35$ range. There were $14.9 \%$ of patients with a mutation frequency $<0.15$, and $55.3 \%$ of patients with a mutation frequency $\geq 0.15$ and $\leq 0.35$. 


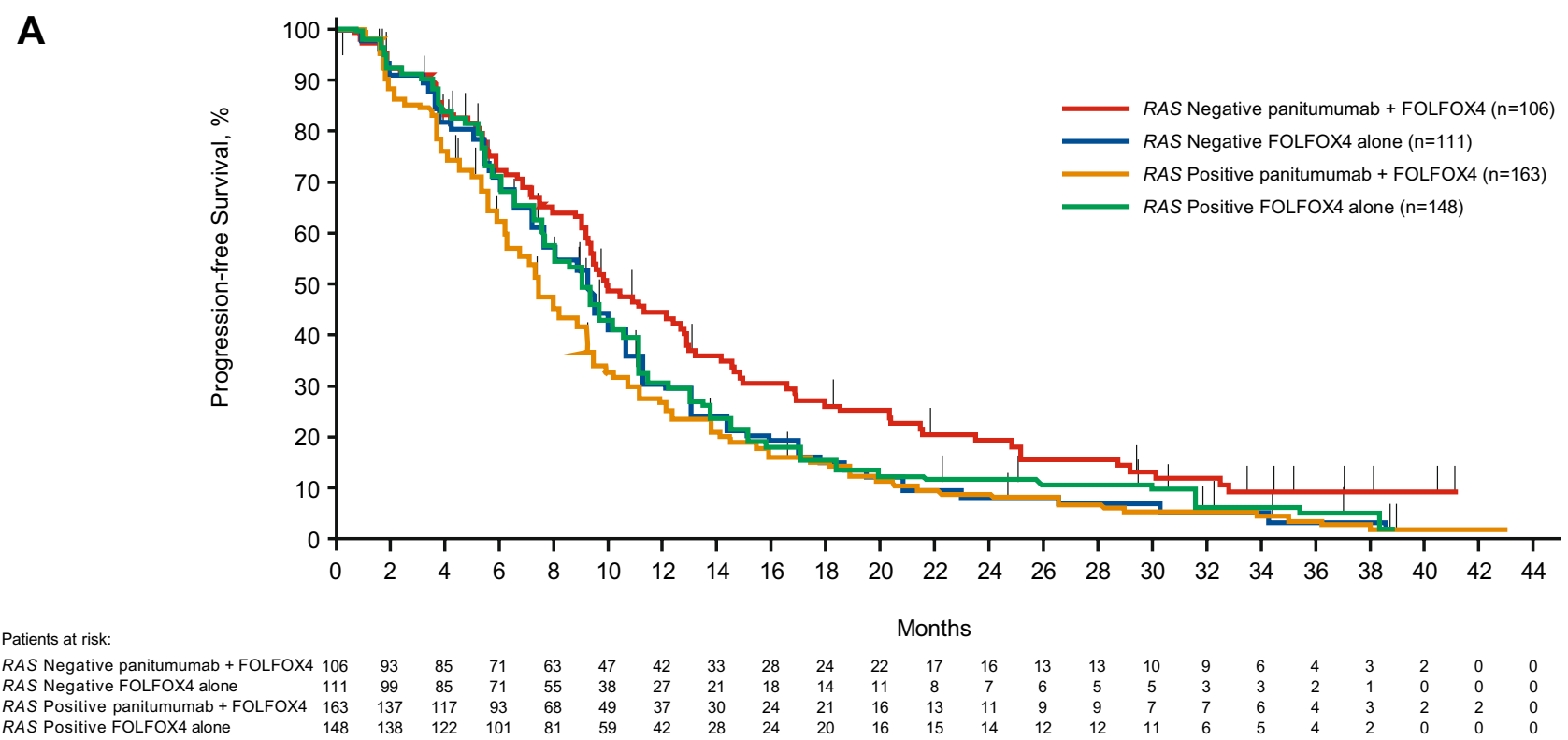

\section{B}

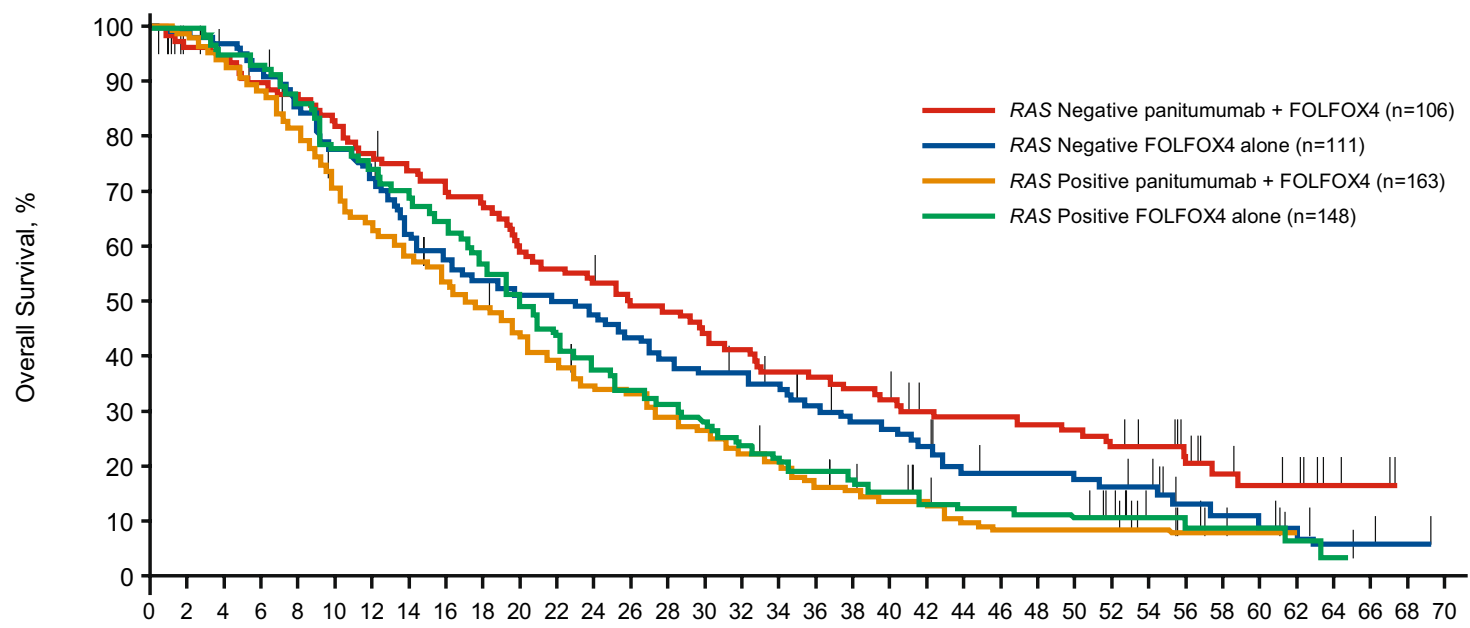

Patients at risk:

RAS Negative panitumumab + FOLFOX4 106 $\begin{array}{ll}\text { RAS Negative FOLFOX4 alone } & 111 \\ \text { RAS Positive panitumumab + FOLFOX4 } & 163\end{array}$ $\begin{array}{ll}\text { RAS Positive FOLFOX4 alone } & 148\end{array}$

$\begin{array}{lcccc}106 & 92 & 79 & 68 & 5 \\ 111 & 98 & 78 & 60 & 5 \\ 163 & 140 & 99 & 76 & 5 \\ 148 & 137 & 107 & 78 & 5\end{array}$

Fig. 2 Kaplan-Meier plots of $\mathbf{A}$ progression-free survival ${ }^{\mathrm{a}}$ and $\mathbf{B}$ overall survival ${ }^{\mathrm{b}}$ according to $R A S$ status and treatment. ${ }^{\mathrm{a}}$ Data cutoff was August 2, 2010. ${ }^{\mathrm{b}}$ Data cutoff was January 24, 2013

and Sanger sequencing revealed that Sanger sequencing had a false positive rate of $11.1 \%$ (possibly caused by misattribution error or poor stringency amplification), a false negative rate of $6.1 \%$, and a limit of detection of 15-20\% (Tsiatis et al. 2010). In contrast, the National Cancer Institute-Molecular Analysis for Therapy Choice (NCI-MATCH) trial performed analytical validation of their NGS targeted assay covering 265 known somatic mutations and determined a sensitivity of $96.98 \%$, a specificity of $99.99 \%$, and a limit of detection (LOD) for single nucleotide variants of $2.8 \%$ (Lih et al. 2017). The Extended RAS Panel includes steps to interrogate both strands of DNA independently as well as provide high depth of coverage. This approach yields increased sensitivity and specificity with an LOD of 5\% (Praxis ${ }^{\mathrm{TM}}$ Extended Panel 2017). This is particularly important when assessing rare mutations that exist in a heterogeneous population of neoplastic cells. In an evaluation of NPA, the NGS panel found mutations in five patients that were categorized as false positives because Sanger did not detect these 
Table 3 Primary efficacy parameters: PFS and OS for $R A S$ Positive

\begin{tabular}{|c|c|c|c|}
\hline \multirow[b]{2}{*}{$\begin{array}{l}\text { Endpoint (Data } \\
\text { Cutoff Date) }\end{array}$} & \multirow[b]{2}{*}{ Parameter } & \multicolumn{2}{|c|}{$K R A S$ Exon 2/RAS Positive } \\
\hline & & $\begin{array}{c}\text { Panitumumab }+ \\
\text { FOLFOX4 } \\
(\mathrm{n}=30)\end{array}$ & $\begin{array}{l}\text { FOLFOX4 Alone } \\
\qquad(n=27)\end{array}$ \\
\hline \multirow[t]{3}{*}{ PFS (2010) } & Events, n (\%) & $26(87)$ & $20(74)$ \\
\hline & $\operatorname{Median}^{\mathrm{b}}(95 \% \mathrm{CI})$ & $9.1(6.2-10.8)$ & $11.1(6.4-13.8)$ \\
\hline & $\mathrm{HR}^{\mathrm{c}}(95 \% \mathrm{CI})$ & \multicolumn{2}{|c|}{$1.477(0.796-2.741)$} \\
\hline \multirow[t]{3}{*}{ OS (2013) } & Events, n (\%) & $25(83)$ & $21(78)$ \\
\hline & Median $^{\mathrm{b}}(95 \% \mathrm{CI})$ & $20.9(14.8-30.5)$ & $22.4(13.0-39.4)$ \\
\hline & $\operatorname{HR}^{\mathrm{c}}(95 \% \mathrm{CI})$ & \multicolumn{2}{|c|}{$1.346(0.728-2.488)$} \\
\hline
\end{tabular}

$\mathrm{CI}=$ confidence interval; $\mathrm{ECOG}=$ Eastern Cooperative Oncology Group; $\mathrm{HR}=$ hazard ratio; $\mathrm{OS}=$ overall survival; $\mathrm{PFS}=$ progression-free survival.

${ }^{\text {aS }}$ Subset of RAS Positive; patients who did not have a mutation in KRAS exon 2 but did have an Extended RAS Panel mutation.

${ }^{b}$ Kaplan-Meier estimate of median time (months) to event; 95\% CI is based on a sign test (Brookmeyer and Crowley, 1982).

${ }^{\mathrm{c}} \mathrm{HR}$ presented as panitumumab plus FOLFOX4:FOLFOX4 alone is estimated from Cox proportional hazards model stratified by region (Western Europe, Canada, and Australia vs rest of world) and ECOG score (0 or 1 vs 2$)$.

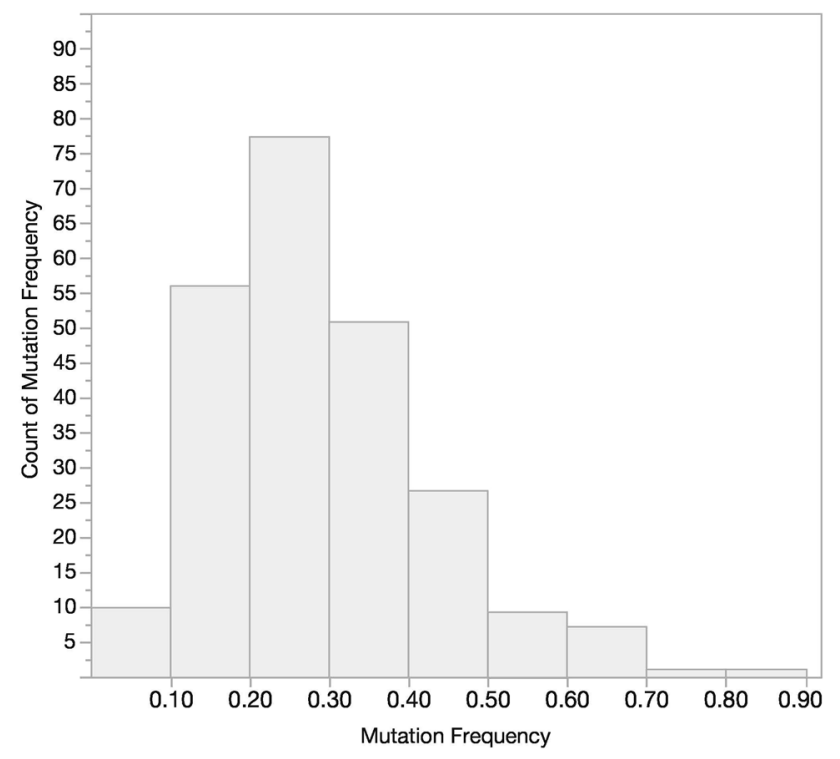

Fig. 3 Extended RAS Panel mutation frequency distribution for positive samples $(n=235)$

mutations. We postulate that the NGS results represented true positives, while Sanger produced five false negatives. Nevertheless, concordance between the NGS assay results and Sanger results was excellent, with a PPA of $98.7 \%$ and an NPA of $97.6 \%$.

Clinical validation of the Extended RAS Panel entailed an analysis of clinical outcomes from the PRIME study (Douillard et al. 2010). A lack of remaining mCRC tissue samples from the original cohort of 1183 randomized patients limited the number of cases for RAS mutational analysis by NGS. Ultimately, 528 samples passed QC measures to make up the evaluable $R A S$ analysis set; $41 \%$ of samples were categorized as $R A S$ Negative and $59 \%$ as RAS Positive. This is consistent with the Douillard et al. (2013) findings of $48 \%$ RAS Negative and 52\% RAS Positive (Douillard et al. 2013). The comparison of the nonparametric Cox proportional hazards model of PFS between RAS Negative patients receiving panitumumab plus FOLFOX4 versus FOLFOX4 alone $(n=217)$ revealed a statistically significant difference of $P=0.02$ (logrank test) with an HR of 0.700 (95\% CI 0.516-0.948). In the $R A S$ Positive cohort, median PFS was 7.4 months with panitumumab plus FOLFOX4 and 9.2 months with FOLFOX4 alone (HR 1.24; 95\% CI 0.976-1.582), suggesting a lack of clinical benefit with panitumumab, and potential detrimental effect of treatment, in this population. Quantitative interaction testing provided further support of the difference in PFS outcomes between RAS Positive and RAS Negative groups $(P=0.0038)$. In addition, the NGS assay produced HRs for PFS that were nearly identical to the PRIME reanalysis using Sanger sequencing with RAS Negative and RAS Positive HRs of 0.72 and 1.31, respectively (Douillard et al. 2013).

Evaluation of median OS revealed that RAS Negative patients treated with panitumumab plus FOLFOX4 lived longer (median, 26.0 months) than those treated with FOLFOX4 alone (23.1 months). The associated HR was 0.754 (95\% CI $0.555-1.024 ; P=0.07)$. In the $R A S$ Positive group, median OS was 17.4 months in the panitumumab plus FOLFOX4 arm compared with 19.6 months with FOLFOX4 alone. Although the HR of 1.156 (95\% CI 0.906-1.476) was not statistically significant, 
the differences observed in favor of chemotherapy alone are aligned with the findings of two large meta-analyses (Sorich et al. 2015; Therkildsen et al. 2014). As with PFS, quantitative interaction analysis confirmed that panitumumab treatment affected RAS Negative and RAS Positive patients differently, with a $P$ value of 0.0323 . Moreover, the PRIME study reported very similar HRs for OS in $R A S$ Negative patients (median OS, 25.8 months versus 20.2 months; HR $=0.77 ; P=0.009$ ) and the RAS Positive group (median OS, 15.5 months versus 18.7 months; $\mathrm{HR}=1.21$; $P=0.001$ ) (Douillard et al. 2013). The lack of statistical significance reported here is likely due to the small sample size in this study ( $n=441$ versus $n=1060$ for the primary analysis), which likely resulted from the lack of remaining samples available and insufficient DNA quality. The low prevalence of individual mutations in KRAS exons 3 and 4 and NRAS exons 2, 3, and 4 range from 0.5 to $6.7 \%$ (Sorich et al. 2015) and help to retain the balance between the $R A S$ Negative and RAS Positive Analysis Sets.

The distribution of mutations found within the KRAS and NRAS exons in this study are similar to those reported in published data (Sorich et al. 2015). Additionally, the distribution of mutation frequencies was also within the expected range given minimum 50\% tumor content.

This analysis compared $R A S$ testing results from Sanger sequencing and from the Extended RAS Panel for patients with mCRC treated with panitumumab. Utilization of this assay is consistent with recent clinical care guidelines regarding RAS testing in mCRC (Sepulveda et al. 2017). Overall, NGS allows for broad, rapid, highly specific analysis of genomic regions of interest, and these results support the use of the Extended RAS Panel as a companion diagnostic for the selection of patients who may derive benefit from panitumumab therapy. Testing for KRAS and NRAS mutations is recommended by both the NCCN and ESMO guidelines for all patients with mCRC; only patients with wild-type $R A S$ tumors are eligible for antiEGFR therapy (National Comprehensive Cancer Network 2017; Van Cutsem et al. 2016).

\begin{abstract}
Acknowledgements The authors would like to thank Meghan Johnson, $\mathrm{PhD}$ (Complete Healthcare Communications, LLC, an ICON plc company, West Chester, PA, USA), whose work was funded by Amgen Inc., for medical writing assistance in the preparation of this manuscript. Amy Mueller, MD, provided clinical expertise and contributed to the writing and review of this manuscript. This study was funded by Amgen Inc. and Illumina, Inc.
\end{abstract}

Funding This study was funded by Amgen Inc. and Illumina, Inc.

\section{Compliance with ethical standards}

Conflict of interest N. Udar and R. Slaughter are former employees of and own stock in Illumina, Inc. D. Vavrek, K. Meier, A. Iyer, and K. Gutekunst are employees of and own stock in Illumina, Inc. C. Lofton-
Day, J. Dong (and spouse), and A. S. Jung are employees of and own stock in Amgen Inc. B. A. Bach is a former employee of and owns stock in Amgen Inc. M. Peeters has received research funding from Amgen Inc. and Roche, and has served on speakers bureaus for Amgen Inc., Sanofi, Servier, and Bayer. J.-Y. Douillard has nothing to disclose.

Ethical approval Samples were obtained from the PRIME study. All patients had given informed consent in the PRIME study, which was approved by responsible ethics committees and was carried out in accordance with the principles of good clinical practice and the Declaration of Helsinki. Details on ethics procedures can be found in the original PRIME study publication (Douillard et al. 2010).

Open Access This article is distributed under the terms of the Creative Commons Attribution 4.0 International License (http://creativeco mmons.org/licenses/by/4.0/), which permits unrestricted use, distribution, and reproduction in any medium, provided you give appropriate credit to the original author(s) and the source, provide a link to the Creative Commons license, and indicate if changes were made.

\section{References}

Allegra CJ, Rumble RB, Hamilton SR, Mangu PB, Roach N, Hantel A, Schilsky RL (2016) Extended RAS gene mutation testing in metastatic colorectal carcinoma to predict response to anti-epidermal growth factor receptor monoclonal antibody therapy: American Society of Clinical Oncology Provisional Clinical Opinion update 2015. J Clin Oncol 34:179-185

Amado RG, Wolf M, Peeters M, Van Cutsem E, Siena S, Freeman DJ, Juan T, Sikorski R, Suggs S, Radinsky R, Patterson SD, Chang DD (2008) Wild-type KRAS is required for panitumumab efficacy in patients with metastatic colorectal cancer. J Clin Oncol 26:1626-1634

Bokemeyer C, Kohne CH, Ciardiello F, Lenz HJ, Heinemann V, Klinkhardt U, Beier F, Duecker K, van Krieken JH, Tejpar S (2015) FOLFOX4 plus cetuximab treatment and RAS mutations in colorectal cancer. Eur J Cancer 51:1243-1252

Douillard JY, Siena S, Cassidy J, Tabernero J, Burkes R, Barugel M, Humblet Y, Bodoky G, Cunningham D, Jassem J, Rivera F, Kocakova I, Ruff P, Blasinska-Morawiec M, Smakal M, Canon JL, Rother M, Oliner KS, Wolf M, Gansert J (2010) Randomized, phase III trial of panitumumab with infusional fluorouracil, leucovorin, and oxaliplatin (FOLFOX4) versus FOLFOX4 alone as first-line treatment in patients with previously untreated metastatic colorectal cancer: the PRIME study. J Clin Oncol 28:4697-4705

Douillard JY, Oliner KS, Siena S, Tabernero J, Burkes R, Barugel M, Humblet Y, Bodoky G, Cunningham D, Jassem J, Rivera F, Kocakova I, Ruff P, Blasinska-Morawiec M, Smakal M, Canon JL, Rother M, Williams R, Rong A, Wiezorek J, Sidhu R, Patterson SD (2013) Panitumumab-FOLFOX4 treatment and RAS mutations in colorectal cancer. N Engl J Med 369:1023-1034

Gagan J, Van Allen EM (2015) Next-generation sequencing to guide cancer therapy. Genome Med 7:80

Gail M, Simon R (1985) Testing for qualitative interactions between treatment effects and patient subsets. Biometrics 41:361-372

Lih CJ, Harrington RD, Sims DJ, Harper KN, Bouk CH, Datta V, Yau J, Singh RR, Routbort MJ, Luthra R, Patel KP, Mantha GS, Krishnamurthy S, Ronski K, Walther Z, Finberg KE, Canosa S, Robinson H, Raymond A, Le LP, McShane LM, Polley EC, Conley BA, Doroshow JH, Iafrate AJ, Sklar JL, Hamilton SR, Williams PM (2017) Analytical validation of the next-generation sequencing assay for 
a nationwide signal-finding clinical trial: molecular analysis for therapy choice clinical trial. J Mol Diagn 19:313-327

Moorcraft SY, Smyth EC, Cunningham D (2013) The role of personalized medicine in metastatic colorectal cancer: an evolving landscape. Therap Adv Gastroenterol 6:381-395

National Comprehensive Cancer Network (2017) NCCN Clinical Practice Guidelines in Oncology: Colon Cancer v1.2017. http://www. nccn.org/professionals/physician_gls/pdf/colon.pdf. Accessed 15 Dec 2016

Patterson SD, Peeters M, Siena S, Van Cutsem E, Humblet Y, Laethem JV, Andre T, Tian Y, Sidhu R, Oliner KS (2013) Comprehensive analysis of KRAS and NRAS mutations as predictive biomarkers for single agent panitumumab (pmab) response in a randomized, phase III metastatic colorectal cancer (mCRC) study (20020408). J Clin Oncol 31:abstr 3617

Peeters M, Douillard J-Y, Van Cutsem E, Siena S, Zhang K, Williams RT, Wiezorek J (2012) Mutant (MT) KRAS codon 12 and 13 alleles in patients (pts) with metastatic colorectal cancer (mCRC): assessment as prognostic and predictive biomarkers of response to panitumumab (pmab) [abstract]. J Clin Oncol 30:383

Peeters M, Oliner KS, Parker A, Siena S, Van Cutsem E, Huang J, Humblet Y, Van Laethem JL, Andre T, Wiezorek J, Reese D, Patterson SD (2013) Massively parallel tumor multigene sequencing to evaluate response to panitumumab in a randomized phase III study of metastatic colorectal cancer. Clin Cancer Res 19:1902-1912

Peeters M, Oliner KS, Price TJ, Cervantes A, Sobrero AF, Ducreux M, Hotko Y, Andre T, Chan E, Lordick F, Punt CJ, Strickland AH, Wilson G, Ciuleanu TE, Roman L, Van Cutsem E, He P, Yu H, Koukakis R, Terwey JH, Jung AS, Sidhu R, Patterson SD (2015) Analysis of KRAS/NRAS mutations in a phase III study of panitumumab with FOLFIRI compared with FOLFIRI alone as secondline treatment for metastatic colorectal cancer. Clin Cancer Res 21:5469-5479

Praxis $^{\mathrm{TM}}$ Extended RAS Panel (2017) Full prescribing information. Illumina, San Diego, CA

Schwartzberg LS, Rivera F, Karthaus M, Fasola G, Canon JL, Hecht JR, Yu H, Oliner KS, Go WY (2014) PEAK: a randomized, multicenter phase II study of panitumumab plus modified fluorouracil, leucovorin, and oxaliplatin (mFOLFOX6) or bevacizumab plus mFOLFOX6 in patients with previously untreated, unresectable, wild-type KRAS exon 2 metastatic colorectal cancer. J Clin Oncol 32:2240-2247

Sepulveda AR, Hamilton SR, Allegra CJ, Grody W, Cushman-Vokoun AM, Funkhouser WK, Kopetz SE, Lieu C, Lindor NM, Minsky
BD, Monzon FA, Sargent DJ, Singh VM, Willis J, Clark J, Colasacco C, Rumble RB, Temple-Smolkin R, Ventura CB, Nowak JA (2017) Molecular biomarkers for the evaluation of colorectal cancer: guideline from the American Society for Clinical Pathology, College of American Pathologists, Association for Molecular Pathology, and American Society of Clinical Oncology. J Mol Diagn 19:187-225

Shendure J, Ji H (2008) Next-generation DNA sequencing. Nat Biotechnol 26:1135-1145

Sorich MJ, Wiese MD, Rowland A, Kichenadasse G, McKinnon RA, Karapetis CS (2015) Extended RAS mutations and anti-EGFR monoclonal antibody survival benefit in metastatic colorectal cancer: a meta-analysis of randomized controlled trials. Ann Oncol 26:13-21

Therkildsen C, Bergmann TK, Henrichsen-Schnack T, Ladelund S, Nilbert M (2014) The predictive value of KRAS, NRAS, BRAF, PIK3CA and PTEN for anti-EGFR treatment in metastatic colorectal cancer: a systematic review and meta-analysis. Acta Oncol $53: 852-864$

Tsiatis AC, Norris-Kirby A, Rich RG, Hafez MJ, Gocke CD, Eshleman JR, Murphy KM (2010) Comparison of Sanger sequencing, pyrosequencing, and melting curve analysis for the detection of KRAS mutations: diagnostic and clinical implications. J Mol Diagn 12:425-432

Van Cutsem E, Lenz HJ, Kohne CH, Heinemann V, Tejpar S, Melezinek I, Beier F, Stroh C, Rougier P, van Krieken JH, Ciardiello F (2015) Fluorouracil, leucovorin, and irinotecan plus cetuximab treatment and RAS mutations in colorectal cancer. J Clin Oncol 33:692-700

Van Cutsem E, Cervantes A, Adam R, Sobrero A, Van Krieken JH, Aderka D, Aranda Aguilar E, Bardelli A, Benson A, Bodoky G, Ciardiello F, D'Hoore A, Diaz-Rubio E, Douillard JY, Ducreux M, Falcone A, Grothey A, Gruenberger T, Haustermans K, Heinemann V, Hoff P, Kohne CH, Labianca R, Laurent-Puig P, Ma B, Maughan T, Muro K, Normanno N, Osterlund P, Oyen WJ, Papamichael D, Pentheroudakis G, Pfeiffer P, Price TJ, Punt C, Ricke J, Roth A, Salazar R, Scheithauer W, Schmoll HJ, Tabernero J, Taieb J, Tejpar S, Wasan H, Yoshino T, Zaanan A, Arnold D (2016) ESMO consensus guidelines for the management of patients with metastatic colorectal cancer. Ann Oncol 27:1386-1422

Vaughn CP, Zobell SD, Furtado LV, Baker CL, Samowitz WS (2011) Frequency of KRAS, BRAF, and NRAS mutations in colorectal cancer. Genes Chromosomes Cancer 50:307-312

\section{Affiliations}

\section{Nitin Udar ${ }^{1}$ (1) $\cdot$ Catherine Lofton-Day ${ }^{2} \cdot$ Jun Dong ${ }^{3} \cdot$ Darcy Vavrek $^{4} \cdot$ A. Scott Jung ${ }^{5} \cdot$ Kristen Meier $^{4} \cdot$ Anita lyer $^{1}$. Ryan Slaughter ${ }^{6} \cdot$ Karen Gutekunst $^{1} \cdot$ Bruce A. Bach $^{7} \cdot$ Marc Peeters $^{8} \cdot$ Jean-Yves Douillard $^{9}$}

1 Department of Clinical Genomics Assay Development, Oncology, Illumina, Inc., 5200 Illumina Way, San Diego, CA 92122, USA

2 Department of Medical Sciences, Amgen Inc., Thousand Oaks, CA, USA

3 Department of Biostatistical Sciences, Amgen Inc., Thousand Oaks, CA, USA

4 Department of Biostatistics, Illumina, Inc., San Diego, CA, USA

5 Department of Research and Development, Amgen Inc., Thousand Oaks, CA, USA
6 Department of Clinical Affairs, Illumina, Inc., San Diego, CA, USA

7 Department of Global Oncology Development, Amgen Inc., Thousand Oaks, CA, USA

8 Department of Oncology, Antwerp University Hospital, Edegem, Belgium

9 Department of Medical Oncology, ICO René Gauducheau, Nantes, France 